\title{
Proporciones generales en las iglesias patrimoniales de Granada, Nicaragua
}

\section{General proportions in the heritage churches of Granada, Nicaragua}

\author{
Luis Carlos Cruz Ramirez ${ }^{1}$, Evelin Cruz Contreras ${ }^{2}$ \\ ${ }^{1}$ Instituto Politécnico Nacional, Miembro ICOMOS México \\ ${ }^{2}$ Grupo DAIEC, México \\ 1luisram06@gmail.com / https://orcid.org/0000-0002-3651-4471 \\ ²evelin.cruz.c@gmail.com / https://orcid.org/0000-0002-8158-3177
}

Recibido el 23 de octubre de 2020, aprobado el 28 de diciembre de 2020

\begin{abstract}
RESUMEN | El objetivo de este artículo es presentar un análisis sobre la proporción en los edificios monumentales religiosos del departamento de Granada, Nicaragua. Los objetos de estudios fueron los edificios religiosos catalogados como patrimonio inmueble, localizados en este departamento. Se consideró como guía para esta investigación los edificios registrados en Catálogos de Bienes Culturales de Granada. Con base en dos catálogos consultados, la muestra obtenida fue de 11 edificios. Dos análisis probabilísticos fueron realizados, considerando las propiedades de la muestra y los objetivos de la investigación: análisis de regresión y análisis de conglomerados. Se encontró que los edificios catalogados de género religioso, localizados en el departamento de Granada, en un solo conjunto no responden a un solo patrón de proporción. En promedio se aproximan a la relación 1: 2.42. Sin embargo, los coeficientes de correlación y determinación son valores medianamente representativos. Los resultados obtenidos son de utilidad para ampliar los conocimientos en ciencia básica en el ámbito del conocimiento de las proporciones mismas, de los edificios locales de este género. Fortuitamente, podrá ser de utilidad en futuros proyectos de proyección de nuevos edificios que deseen considerar este aspecto formal, adoptando el criterio de diseño tipológico..
\end{abstract}

PALABRAS CLAVE | Arquitectura, Diseño, Iglesias, Patrimonio, Proporciones

ABSTRACT I The aim of this paper is to present an analysis on general proportions in the
heritage churches of Granada, in Nicaragua. Cases of studies were religious building catalogued
as heritage asset, located in this geographic zone. Building recording and enrolled in Catalogues
of Cultural Assets were considered in this research. Based on two consulted catalogues, the
sample obtained was of 11 buildings. Two probabilistic analyses were carried out, considering
the properties of the sample and the objectives of the investigation: regression analysis and
cluster analysis. It was found that the buildings classified as religious, located in the department
of Granada, in a single group do not respond to a single pattern of proportion. On average, they 
approximate the ratio $1: 2.42$. However, the correlation and determination coefficients are fairly representative values. The results obtained are useful to expand knowledge in basic science in the field of knowledge of the proportions themselves, of local buildings of this kind. Fortuitously, it may be useful in future projection projects for new buildings that wish to consider this formal aspect, adopting the typological design criterion.

KEYWORD | Architecture, Churches, Design, Heritage, Proportion

\section{Introducción}

La forma, característica física o apariencia exterior reconocible de los objetos (Ching, 2005/2012, p. 34), ha sido una de las propiedades mejores cuidadas por los arquitectos al momento de diseñar sus edificios. Al ser una propiedad perceptible, le da el carácter artístico y distintivo al edificio. A su vez, es producto de la creatividad atribuida del diseñador.

Para el diseño de la forma, existen un conjunto de propiedades fundamentales que es necesario considerar. Entre estas se puede mencionar la geometría, la simetría y la proporción. La proporción, matemáticamente, es una relación de igualdad que existe entre dos razones, que sirve para comparar dos magnitudes (Miller et al. 2005, p. 341). Se indica por medio de una expresión “A: B”, la cual se enuncia como A es B. En arquitectura, la proporción se conceptualiza como una propiedad de la forma que describe la relación de una parte con el todo (Ching, 2007/2014, p. 306). La relación de proporción entre ancho y largo de un edificio es de 1:5, significa que la longitud es cinco veces la anchura. Esto se utiliza para describir la relación de dimensiones de largo y ancho en proyecciones de alzados y plantas.

El estudio de la proporción ha sido fundamental en el diseño arquitectónico durante toda la historia de la construcción. Es quizás una de las propiedades formales de mayor relevancia, porque atribuye otras características como el orden, la armonía, la unidad, así como valores estéticos (Llorente, 2000, p. 239). Los antecedentes de esta práctica tienen sus primeras manifestaciones que datan de los tiempos de la antigua civilización griega ${ }^{1}$. También se encuentra documentada por Vitruvio $(27 / 1741)^{2}$-primer texto de arquitectura del cual se tiene registro en la actualidad-.

No obstante, las formas arquitectónicas analizadas fuera del academicismo y el diseño canónico, están también relacionadas con la ubicación geográfica, elementos materiales del entorno y un conjunto de factores culturales, tales como: las creencias, aspiraciones, simbolismos y arraigos -entre otros factores- (Rapoport, 1972). Entre las creencias relacionadas con la proporción, se puede mencionar que: (a) en la antigüedad se utilizaba para reproducir y lograr la analogía de las construcciones con formas naturales y formas humanas (Well, 2010; Hiscock, 2007), (b) durante la edad media sirvió para desarrollar formas complejas, (c) en el renacimiento se creía que, si se adoptaban proporciones naturales, entonces se le otorgaba el mismo atractivo estético -bellezaconsiderando la proporción cualidad de perfección (Llorente, 2000, pp. 143, 239). También, el principio de la estabilidad constructiva fue inferido por medio de principios de proporción (Mark, 1993/2002, p. 32).

Las proporciones han sido especialmente cuidadas en edificios representativos en la historia

1 Se pueden encontrar relaciones en los libros de Euclides, descritos y traducidos por Fray Lorenzo de San Nicolas. La proporción fue una temática atractiva entre los matemáticos, que asociaban las series numéricas con relaciones de proporción.

2 Dedica el segundo capítulo del libro VI a las proporciones de los edificios. 
de la construcción. Diferentes tratadistas tales como Alberti (1485), Palladio (1570/2008), Serlio (1547) y Fray Lorenzo de San Nicolas (1663, p. 271) incluyeron en sus tratados secciones que abordaban esta propiedad. Serlio (1547), siguiendo la tradición constructiva occidental, en la cual los edificios religiosos comúnmente han sido proyectados siguiendo estrictas reglas de proporción, presentes en el libro $\mathrm{V}$, sobre lineamientos de diseño de la arquitectura sacra.

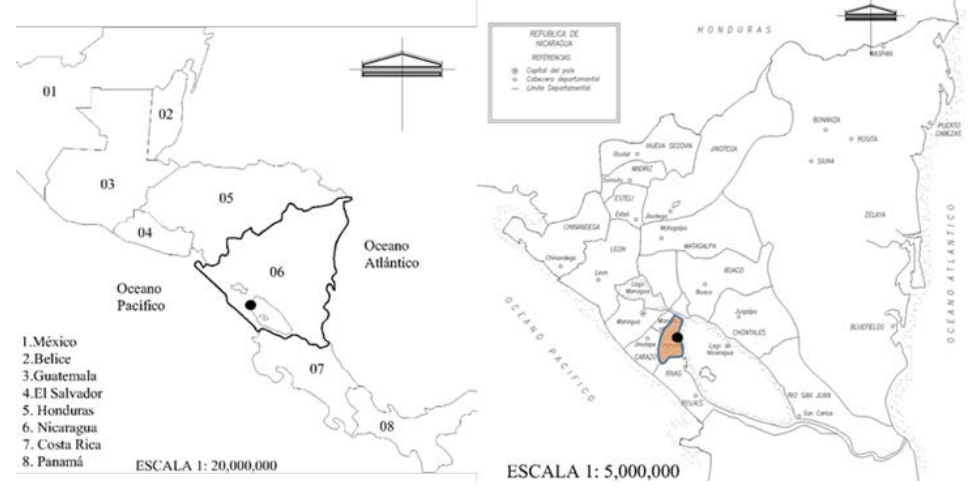

Figura 1: Localización del Departamento de Granada. Elaborado por los autores

En Nicaragua, existen diferentes edificios religiosos patrimoniales en todo el país, construidos desde el siglo XVI hasta el siglo XX. El departamento de Granada (ver Figura 1), es una de las regiones política-administrativa, que posee importantes ejemplos representativos de arquitectura religiosa internacionalmente conocidos - tales como la iglesia la Merced, la iglesia de Xalteva, la iglesia del convento San Francisco y Catedral de Granada-. La Ciudad de Granada, en su casco histórico, posee siete de estos monumentos (ver Figura 2). Pero ¿Existe alguna regla de proporción claramente identificable en los edificios religiosos de Granada? Y si existe, ¿Qué proporciones siguen sus edificios? En caso de no seguir un solo patrón, ¿Es posible identificar conjuntos de edificios que conserven características similares?
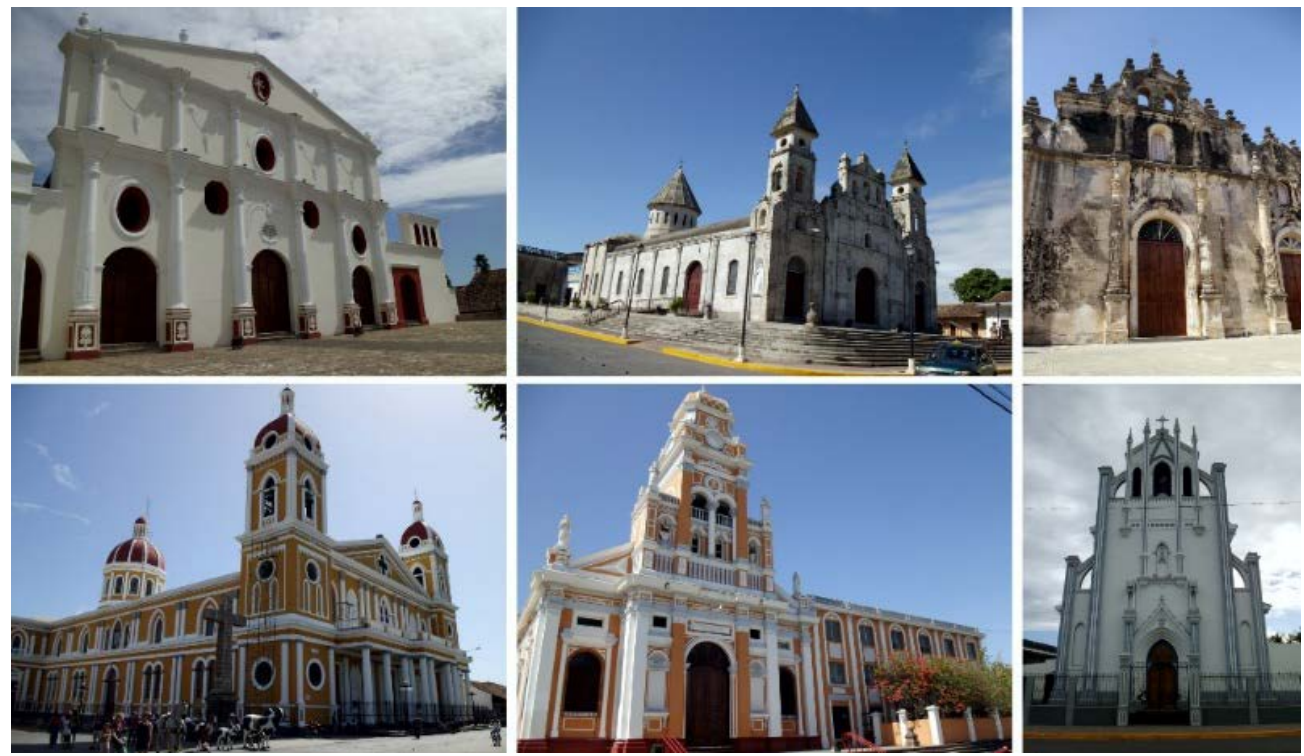

Figura 2: Ejemplares de la arquitectura religiosa patrimonial en la Ciudad de Granada, Nicaragua. De izquierda a derecha en la fila superior: Iglesia del convento San Francisco, Iglesia de Guadalupe, Iglesia de la merced. De izquierda a derecha en la fila inferior: Catedral de Granada, Iglesia de Xalteva y Capilla María Auxiliadora. Elaborado por los autores. 
El objetivo de este artículo es indagar sobre la proporción en planta que siguen los edificios monumentales del departamento de Granada, Nicaragua ${ }^{3}$. De momento, no existe evidencia histórica encontrada que indique precisamente la proporción real aplicada en los edificios religiosos de Granada, Nicaragua, ni en la región centroamericana. Por lo anterior, la conjetura formulada, es que, si bien puede que no todos los edificios sigan la misma dimensión, es probable que estos edificios se aproximen a las reglas establecidas durante el renacimiento europeo, que llegaron a Nicaragua durante el siglo XVI, por las misiones franciscanas -como indica Ovando (2013) - y que fueron las que se replicaron durante siglos. La regla mejor conocida entre los constructores que llegaron a América, fue la regla de proporción de Hontañón de uno es a cinco (1:5), con la recomendación de cincuenta metros de largo y diez metros de ancho ${ }^{4}$ (Kubler, 1984/2008, p. 301). En este contexto, esta investigación se realizó para conocer las proporciones existentes en los edificios religiosos de Granada, tomando como punto de partida la conjetura anterior ${ }^{5}$. Los resultados de esta investigación, en conjunto con otros estudios de este género, pueden ser de utilidad para poder describir las características de la arquitectura religiosa patrimonial nicaragüense. En la siguiente sección se explica al detalle el método que se siguió para poner comprobar o desaprobar la conjetura anterior.

\section{Método}

Primero se determinaron las variables de análisis, seleccionadas a partir de las dos dimensiones básicas - largo y ancho- de su proyección en planta. Cómo objeto de estudios se tomaron los edificios religiosos del departamento de Granada, Nicaragua. El criterio de elegibilidad, fue seleccionar aquellos edificios localizados en el departamento de Granada, Nicaragua que han sido registrado en catálogos oficiales de monumentos históricos, tales como el Catálogo de Bienes Culturales publicado por INC (2011) y Alcaldía de Granada et al. (2004). La población encontrada fue de 12 iglesias. La muestra fue seleccionada de forma determinista ${ }^{6}$, con un total que corresponde a 11 (91\%) edificios existentes publicados en catálogos oficiales de edificios patrimoniales.

Una vez definida la muestra, los datos fueron extraídos de la información publicada en los catálogos existentes. Los datos asignados a las variables son escalares. La fuente de datos son los edificios, pero al tratarse de un meta-análisis, estas dimensiones fueron extraídas de los registros documentales antes mencionados. Las medidas fueron obtenidas manualmente a partir de las proyecciones oficiales publicadas. Estas mediadas también fueron comparadas con proyecciones planas extraídas de bases de datos cartografías digitales disponibles en línea. Estos datos fueron registrados, clasificados y ordenados de forma tabular para posteriormente conducir los análisis. No se realizaron manipulaciones experimentales, ya que no fueron necesarias por el perfil de la investigación ${ }^{7}$.

3 Por esta razón se buscarán datos empíricos, para conocer la realidad.

$4 \mathrm{Si}$ bien no se ha encontrado evidencia histórica, que precisamente indique el uso algún tipo de reglas de proporción en la arquitectura monumental nicaragüense, las reglas de Hontañón podrían ser las más probables. Primero, porque la ciudad y sus edificios fueron trazados desde el siglo XVI, y al igual que en el resto de la Nueva España, también es probable que se hubieran utilizado los mismos lineamientos de construcción para este género religioso, ya que estos desarrollos fueron coordinados por las mismas misiones de padres franciscanos. Los religiosos, en su formación incluían conocimientos de construcción y diseño arquitectónico. Además, se pueden mencionar las conexiones existentes entre la familia de Hontañón en Salamanca y los frailes que llegaron a América.

5 Si la conjetura anterior no es correcta, además de rechazar la hipótesis, los resultados serán siempre de utilidad ya que se logrará tener una aproximación empírica a las proporciones de los edificios.

6 La muestra fue seleccionada de forma determinista, que corresponde al total de los edificios existentes publicados en catálogos oficiales de edificios patrimoniales. La población fue de 6

7 Por el contrario, únicamente se realizaron registros de observaciones encontrados por meta-análisis. 
Debido a que la muestra obtenida, no cumple con el criterio de dimensión de las muestras paramétricas, se optó por analizar los datos por medio de dos modelos que no son sensibles a este criterio: (a) análisis de regresiones y (b) análisis de conglomerados. Los modelos de regresión no requieren distribución normal de los datos ni homogeneidad en las variables (Guisande, Vaamonde y Barreiro, 2011/2013, p. 453). Los modelos de regresiones son de utilidad en este estudio debido a que dicha prueba probabilística sirve para establecer la relación entre dos variables -en el caso de las regresiones lineales- o más variables -en el caso de regresiones múltiples- (Kazmier, 1978/1985). El uso de modelos de regresión podría parecer innecesario, ya que, si se existiera a la perfección la relación de proporción, al aumentar el ancho aumenta el largo, conservándose constante la relación: escenario en el cual se mostraría una recta de regresión con valores constantes, con un $\mathrm{r} 2=1$. Pero existen varias razones por las cuales es improbable que la relación largo-ancho se cumpliera a cabalidad. En principio, porque el escenario de relaciones de proporción largo-ancho constantes, es un escenario hipotético-ideal, ya que los procesos de construcción en la realidad tienden a ser imprecisos, porque en la mayoría de las construcciones realizadas en siglos anteriores fueron ejecutadas con materiales no-estandarizados, con usencia de herramientas de alta precisión en el diseño y la construcción, diferentes a los que pueden existir en la construcción del siglo XXI en el primer mundo. Lo más probable sería obtener resultados, próximos a esta proporción, con un ruido generado en la dispersión, por los errores propios de la naturaleza constructiva, que deberían de ser al menos perceptibles en una gráfica de dispersión. Debido a que también se desconocen tales errores, este estudio podría ser un primer acercamiento a conocer este rango de dispersión.

La hipótesis nula (Ho) planteada es que no existe una relación de proporción 1:5 en los edificios religiosos patrimoniales de Granada. La hipótesis alternativa (Hi) planteada es que si existe dicha relación de proporción. La recta de regresión también es de utilidad para poder conocer la proporción probable de cualquier otro edificio monumental, a la fecha no documentado, que esté dentro del rango de proporciones analizadas. En este caso se aplicaron para establecer la relación de la variable longitud con la variable anchura, con la cual conocer una función que describa en promedio como es la proporción de los edificios seleccionados. Los análisis fueron realizados utilizando algoritmos de probabilidad computacional ejecutados en $\mathrm{R}$ programming, usando las bibliotecas stats -para el análisis y gráfica- y ggplot2 -para obtener otras ilustraciones gráficas(para revisión de procedimientos detallados revisar Wickham (2016) y Kassambara (2013)).

En una función de regresión lineal, $\mathrm{y}=\mathrm{mx} \pm \mathrm{b}$, la pendiente $(\mathrm{m})$ de la recta, indica la cantidad de veces que se incrementa el valor de " $y$ " en función de "x", y el valor "b", llamado inserción, el cual indica el valor de " $y$ " cuando " $x$ " es igual a cero. Para efectos de esta investigación, si " $y$ " es el largo, que está en función del ancho " $x$ ", la pendiente de la función indica la relación de proporción que se busca conocer. Es decir que, bajo la hipótesis mencionada en la sección anterior, se esperaría un valor de "m" igual o muy cercano a cinco y un "b" igual o cercano a cero. Así al sustituir la función $y=m x+b$, se reduciría a $y=5 x+0$, que indicaría que, por cada valor de la anchura " $x$ ", la longitud " $y$ " se incrementa cinco veces. Por el contrario, si el valor es diferente de cinco, la pendiente servirá para conocer la relación de proporción existente en la realidad.

Posteriormente, se procedió a conocer los índices de correlaciones y determinaciones para conocer la relación entre las variables. Los parámetros de utilidad fueron los coeficientes de correlación (R) que indican con valor escalar la relación entre las dos variables, así como el coeficiente de determinación (R2) que es de utilidad para conocer el grado de determinación. La correlación es significativa cuando su rango se encuentra en los cuartos extremos del rango del valor de $\mathrm{r}(-0.5$ $>$ r > 0.5) (Lind et al., 2008/2012). Debido a que la correlación varía según la cantidad de datos 
analizados, la condición $-0.5>\mathrm{r}>0.5$ sólo es válida cuando existen un rango de 7-9 datos. $\mathrm{Si}$ son menos datos, se requiere mayor aproximación a los límites. Si hay más de nueve datos, se aceptan valores menores al rango antes enunciado. Por tanto, se procedió a calcular la función de probabilidad t de Student para conocer el valor exacto necesario de $\mathrm{r}$ de Pearson, para afirmar la existencia de correlación en función de la cantidad de datos. El valor t de Student, se calcula con la siguiente expresión descrita por Triola (2013), según sea la r de Pearson.

$$
t=\frac{r \sqrt{n-2}}{\sqrt{1-r^{2}}}
$$

Por otro lado, el análisis de conglomerados -también conocido como análisis de clúster- es una prueba útil para agrupar observaciones a partir de las similitudes encontradas en las características de las variables analizadas (Hair, Anderson, Tatam, \& Black, 1999, p. 492), se aplican en estudios exploratorios, donde las observaciones aparentemente no tienen patrones, a menos que se analicen por grupos separados. Los criterios de análisis utilizados fueron los siguientes: (a) el criterio de distancia, utilizado para conocer las observaciones más próximas, fue por el método de la distancia euclídea - la distancia geométrica medida entre dos puntos descrita por la función punto pendiente-, (b) el criterio para aglomeración utilizado para crear los grupos entre las observaciones más próximas, fue el método de distancias Ward, que es el método de la suma de los cuadrados de distancias de cada objeto, en el cual, en cada paso une dos elementos -algoritmo que reduce perdida de información-, (c) se utilizó el método del codo para conocer el parámetro " $k$ " para conocer el número de grupos significativos por agrupar -parte los modelos K-Means-, el cual fue contrastado con el criterio clásico de selección de análisis de clústers -la mayor distancia lateral obtenida y visible en el dendrograma ${ }^{8}$ no cortada en paralelo por otra rama del árbol. Estos resultados se muestran gráficamente en un dendrograma. Antes de hacer el análisis se realizó la normalización de los datos para ajustar las escalas. Todos estos criterios han sido ampliamente documentados en la literatura de probabilidades -por ejemplo, Guisande et al. (2011/2013), Hair et al. (1999), James, Witten, Hastie, \& Tibshirani (2017) y Paradis (2012)-.

\section{Resultados}

Al analizar las proporciones encontradas de forma individual, se logró conocer que no existe una regla de proporción que responda a un solo patrón de proporción. Estas varían en un rango que va de 1 es a 1.86 hasta 1 es a 4.54. Estas proporciones tampoco siguen un patrón de localización geográfica, ni tiene asociación con la fecha de construcción -patrón temporal-. Al contrastar las observaciones en la Figura 3, el conjunto de datos encontrados -representados con puntos- contra la hipótesis alternativa -representados por triángulos-, se observa claramente las diferencias, razón por la cual se afirma que, el conjunto de edificios religiosos catalogados localizados en el departamento de Granada no sigue en términos probabilísticos el patrón de proporción de 1:5. Se acepta la Ho y se rechaza Hi.

8 Dendrograma es la representación de los grupos por medio de un diagrama de árbol. 


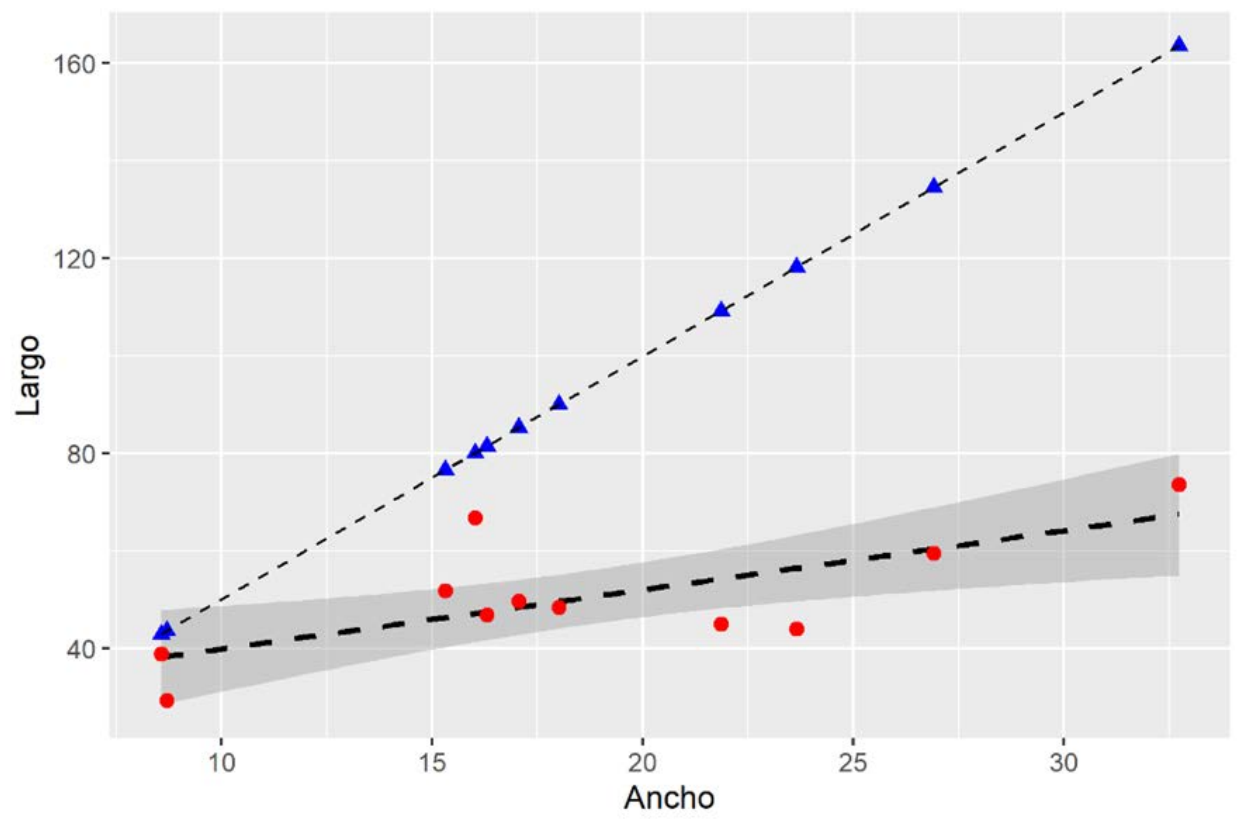

Figura 3: Diagrama de dispersión y modelo de regresión de las proporciones de los edificios religiosos de Granada. Elaborado por los autores.

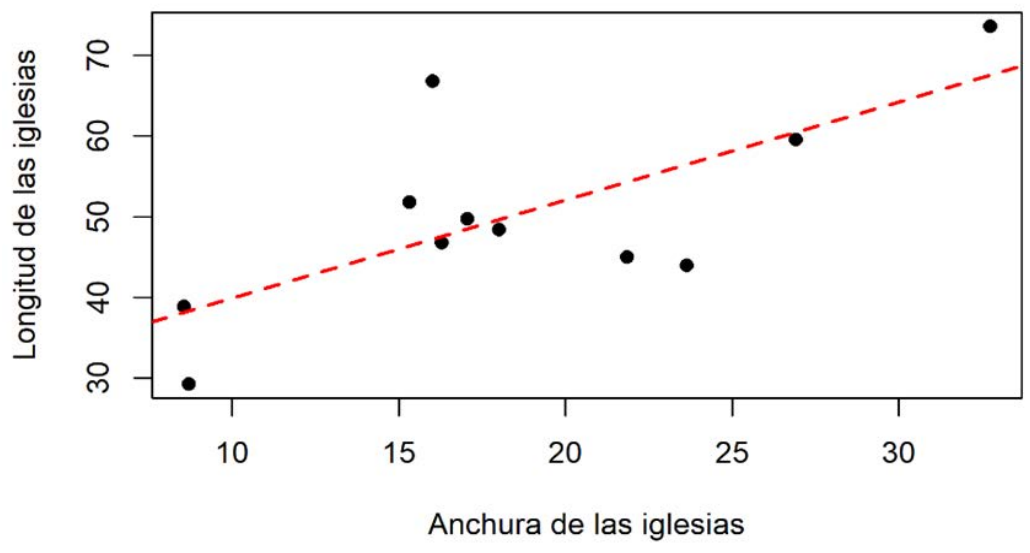

Figura 4: Diagrama de dispersión y modelo de regresión de las proporciones de los edificios religiosos de Granada. Elaborado por los autores.

El modelo de regresión encontrado que describe el conjunto de edificios religiosos del departamento de Granada, es $y=1.2132 x+27.741$, donde " $y$ " es el largo y " $x$ " es el ancho de la iglesia. El coeficiente de correlación $(r=0.7067)$ se encuentra dentro de un rango relativamente aceptable ${ }^{9}$. Sin embargo, su capacidad de predicción es relativamente baja, ya que sólo se puede predecir el $70 \%$ de los casos. El coeficiente de determinación $\left(\mathrm{r}^{\wedge} 2=0.4995\right)$ no es verdaderamente eficiente (Figura 4).

La expresión que puede facilitar dicha relación, se encontró al evaluar el modelo de manera 
inversa ${ }^{10}$, considerando la variable de predicción "y" como el ancho, en función de la longitud, ya que se trata de una relación bilateral (ver Figura 4). El resultado obtenido es mucho más fácil de comprender que el anterior, ya que el modelo de regresión encontrado fue $\mathrm{y}=0.4117 \mathrm{x}-2.0918$. Esta expresión rescrita, es $x=2.429 y+5.089$. Esto indica que la función no parte del origen, sino que su intersección con el eje de las ordenadas, en el punto cero, está a cinco unidades hacia arriba del origen y que la proporción promedio es de 1:2.42. No obstante, los coeficientes de correlación y determinación son los mismos que los mencionados en el párrafo anterior.

Adicionalmente, se analizó la normalidad de los residuos (Figura 5) para conocer la significación del modelo. Se encontró que las proporciones de la Iglesia María Auxiliadora es un dato atípico (dato N10). Si se obviara este dato, los coeficientes de correlación y determinación mejorarían $\left(\mathrm{r}^{\wedge} 2=0.72\right.$ y $\left.\mathrm{r}=0.85\right)$, sin embargo, la relación de proporción media sería de $1: 1.82$, con una intersección en el eje de las ordenadas en 7.73. Esta proporción continúa siendo muy distante a la hipótesis de 1:5.

Para una descripción más precisa del fenómeno, se procedió a indagar si era posible agrupar los diferentes edificios en función de las dos medidas analizadas en esta relación de proporción. Para esto, primero se indagó sobre la cantidad óptima de grupos (k optimo) por el método del codo. En la Figura 6, se presenta como varía la suma de los cuadrados dentro de cada grupo -por sus siglas en inglés wcss- en el eje de las ordenadas y en el eje de las abscisas el número de grupo.
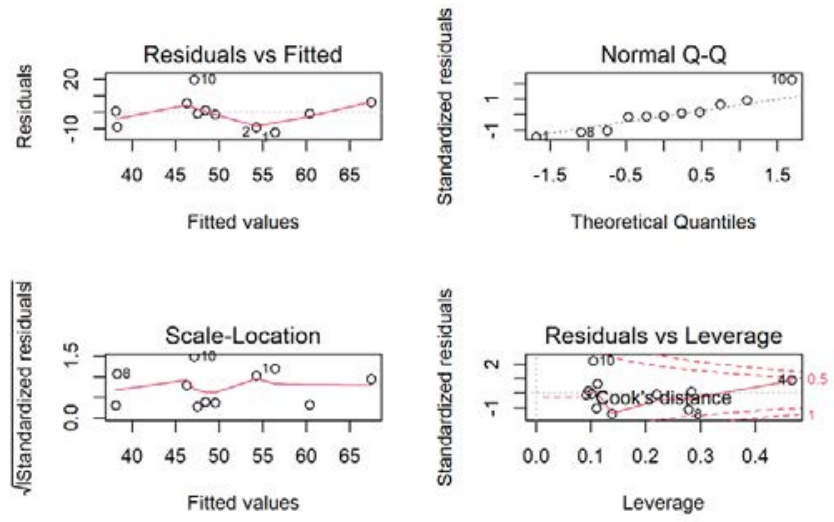

Figura 5: Análisis de residuos del modelo de regresión. Elaborado por los autores.

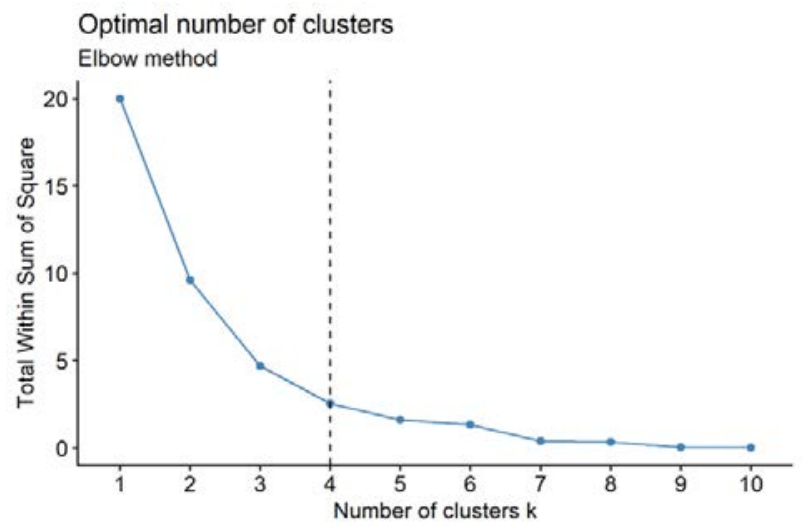

Figura 6: Estimación de los k grupos significativos. Elaborado por los autores.

10 Esto sólo se puede hacer si existe una relación bilateral. Debido a que el fenómeno que se estudia cumple con esta condición se procedió a analizarlo de esa manera. 
El valor k seleccionado fue cuatro (4), con el cual se procedió a realizar el análisis de los conglomerados, para conocer las posibles agrupaciones. El resultado de este análisis se presenta en la Figura 7, por medio de un dendrograma. Los clústeres obtenidos se presentan en la Tabla 1, junto con otra agrupación generada de manera determinista en función de los rangos de proporción individuales, considerando valores medios de proporciones ${ }^{11}(\mathrm{vmp})$ siguientes 1:2, 1:3 y 1:4. Estos grupos a su vez se presentan graficados en las Figura 8 y Figura 9.

Clasificación de los edificios religiosos catalogados

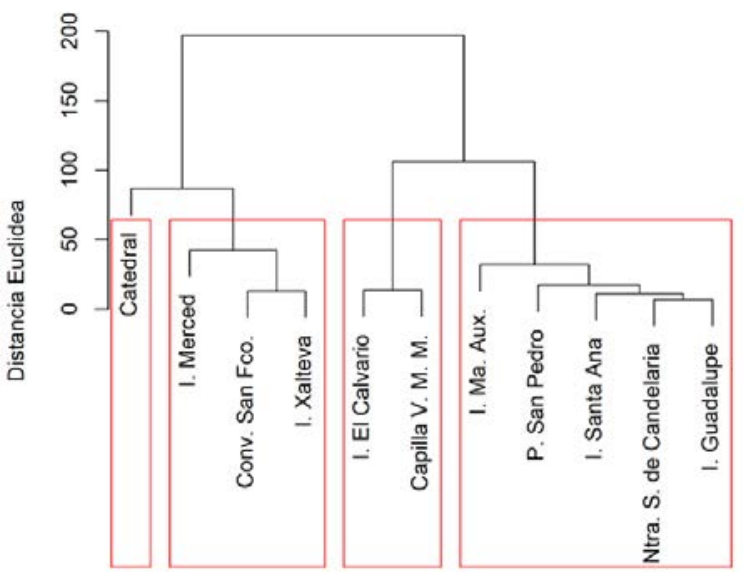

Edificios religiosos catalogados Método de aglomeración: Ward D2

Figura 7: Dendrogama de los conglomerados de edificios según sus proporciones y año de construcción. Elaborado por los autores.

Tabla 1: Agrupaciones de edificios religiosos catalogados.

\begin{tabular}{llll}
\hline Edificios religiosos catalogados & Clúster* & vmp** & Época de construcción (t) \\
\hline Catedral Granada & A & A & XVI*** \\
Iglesia Merced & B & A & XVI \\
Iglesia María Auxiliadora & B & C & 1922 \\
Iglesia Xalteva & B & A & $1751^{* * *}$ \\
Iglesia el Calvario & C & B & 1840 \\
Capilla Virgen Medalla Milagrosa & C & C & 1930 \\
Parroquia San Pedro de Diriá & D & B & 1739 \\
Iglesia San Francisco & D & A & XVI \\
Iglesia Santa Ana & D & B & 1858 \\
Parroquia Nuestra Señora de Candelaria & D & B & 1795 \\
Iglesia Guadalupe & D & B & XX \\
\hline
\end{tabular}

Nota: ${ }^{*}$ Los clúster tienen validez pirobalística. ${ }^{* *} \mathrm{vpm}=$ valores medios de proporción. ${ }^{* * *}$ Reconstrucciones sobre la misma planta realizadas en el siglo XIX. Estos grupos no-probabilistas fueron elaborados para contrastarse con el modelo probabilista del clúster, formados a partir de medias de proporciones individuales, bajo el siguiente criterio determinista: E (1.5-2.5), F (2.-3.5), G (.5-4.5).

11 Estos se agruparon bajo el siguiente criterio: E (1.5-2.5), F (2.-3.5), G (.5-4.5). 


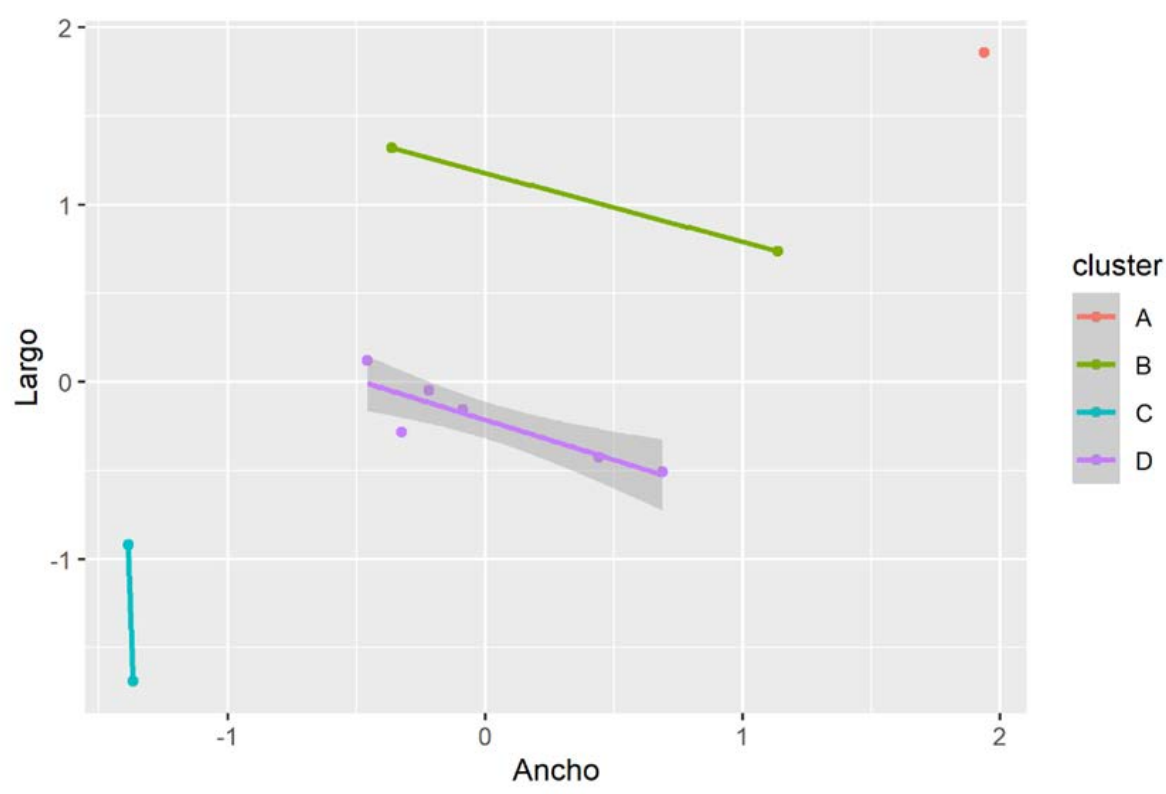

Figura 8: Gráfica de los grupos obtenidos por análisis de conglomerados (clúster). Elaborado por los autores.

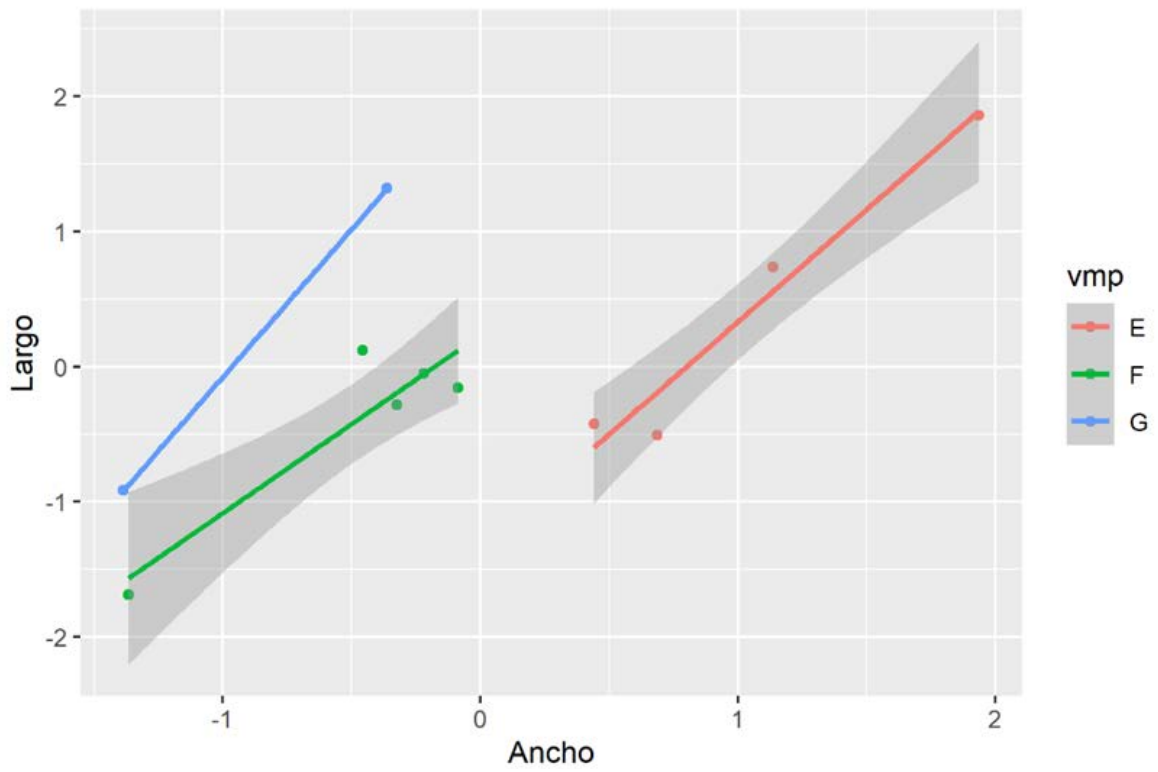

Figura 9: Gráfica de grupos obtenidas a partir de valores medios de proporción. Elaborado por los autores.

En el dendrograma, se observa que, aunque los edificios han sido agrupados considerando sus distancias euclidianas, los grupos obtenidos se podrían describir en función de sus longitudes en planta. El edificio de Catedral (A) se encuentra aislado del resto, esto debido a que tiene las dimensiones más grandes. Sólo se podría agrupar, en nivel de jerarquía superior de clasificación con el segundo grupo (B) encontrado que está compuesto por la Iglesia la Merced y la iglesia María Auxiliadora, que su única relación es que tienen longitudes más grandes registradas. El siguiente conjunto de edificios (D) está compuesto por seis edificios cuyas longitudes varían entre 51-44 m y las anchuras entre 15 y $23 \mathrm{~m}$. El último grupo (C) está compuesto por dos edificios que tienen las menores dimensiones en ambas variables (Figura 8). Por otro lado, la segunda agrupación realizada de manera determinista entre las proporciones observadas (Figura 9), se pudieron agrupar en tres 
conjuntos los cuales conservan las proporciones del modelo de regresión general presentado en la Figura 4.

\section{Conclusiones}

La proporción de los edificios religiosos catalogados como patrimonio localizados en la región de Granada no siguen un patrón de proporción. Aunque los coeficientes de correlación y determinación en conjunto no resultan significativos, luego de realizar la segmentación por el método de conglomerados, se pudo conocer que al menos existen cuatro patrones de proporciones identificables.

En este estudio se propuso una forma probabilística de poder estimar las proporciones de los edificios, basadas en datos. Es una forma no convencional, pero fácilmente aplicable que está sustentada en teoría probabilística. Uno de los problemas que surgen al aplicar este tipo de análisis está relacionado con la capacidad de poder obtener datos de los edificios, ya que no resulta fácil tener acceso a ellos o poder tomar medidas apropiadas.

Entre los problemas abiertos se pueden enunciar los siguientes: ¿Qué proporciones existen en los edificios religiosos en otras regiones?, ¿Qué otras relaciones de proporción pueden indagarse?, ¿Existen patrones entre diferentes edificios localizados en diferentes regiones que puedan agruparse por sus características comunes? Como queda indicado en las preguntas anteriores, este trabajo puede replicarse en otras regiones geográficas, para encontrar posibles patrones de proporción o patrones de agrupación. También puede extenderse a otras relaciones de proporción, que sirvan para poder entender la arquitectura regional. Es necesario considerar, que eventualmente, al replicar este modelo de análisis podrían encontrarse variaciones por causa de datos atípicos (outlier), para lo que deberá de tratarse con técnicas probabilísticas apropiadas.

\section{Bibliografía}

Alberti, L. B. (1485). De re aedificatoria. Firenze.

Alcaldía de Granada | Agencia Española de Cooperación Internacional para el Desarrollo (AECID) | Junta de Andalucía. (2004). Catálogo de Bienes Patrimoniales. Granada [Nicaragua]. (A. Reyes, \& J. R. Moreno García, Edits.) Granada, Nicaragua: Oficina del Centro Histórico de Granada $\mid$ Alcaldía de Granada | AECID.

Ching, F. D. (2007/2014). Form, space and order. New Jersey: John Wiley and Sons.

Guisande González, C., Vaamonde Liste, A., \& Barreiro Felpeto, A. (2011/2013). Análisis multivariante: Métodos de clasificación. En Tratamiento de datos estadísticos con R, STATISTICA, SPSS. (págs. 759-809). Editorial DIAZ DE SANTOS.

Guisande González, C., Vaamonde Liste, A., \& Barreiro Felpeto, A. (2011/2013). Regresiones. En Tratamiento de datos estadísticos con R, STATISTICA, SPSS. (págs. 453-482). Editorial DIAZ DE SANTOS.

Hair, J. F., Anderson, R. E., Tatam, R. L., \& Black, W. (1999). Análisis multivariante. Madrid: Prentice Hall.

Hiscock, N. (2007). The Symbol at Your Door. London: Routledge. doi: https://doi. 
org/10.4324/9781315236872

Instituto Nicaragüense de Cultura. (2011). Catálogo de Bienes Culturales Inmuebles de los departamentos de Granada y Masaya. Granada y Masaya: Instituto Nicaragüense de Cultura.

James, G., Witten, D., Hastie, T., \& Tibshirani, R. (2017). An Introduction to Statistical Learning with Applications in R. Springer. doi:https://doi.org/10.1007/978-1-4614-7138-7

Kassambara, A. (2013). ggplo2: Guide to create beautiful graphics in R. STHDA.

Kazmier, L. J. (1978/1985). Estadísticas Aplicadas a la Administración y a la Economía. (C. Esguerra, \& C. De Barella, Trads.) México, D.F.: McGraw Hill.

Kubler, G. (1983/2009). Arquitectura Mexicana del siglo XVI. México: Fondo de Cultura Económica.

Lind, D., Marchal, W., \& \& Wathen, S. (2008/2015). Estadística Aplicada a los Negocios y la Economía (16 ed ed.). (M. D. Obón León, \& J. León Cardenas, Trads.) México, D.F: McGraw Hill.

Llorente Díaz, M. (2000). El saber de la arquitectura y de las artes: la formación de un ámbito de conocimiento desde la antigüedad hasta el siglo XVII. Barcelona: Ediciones UPC; Univ. Politècnica de Catalunya.

Mark, R. (1993/2002). Tecnología arquitectónica hasta la revolución científica. Arte estructura de las grandes construcciones. (J. M. Gómez Acosta, \& D. López Martínez, Trads.) Madrid: Akal.

Miller, C. D., Heeren, V. E., \& Hornsby, J. (2005). Matematica: Razonamiento Y Aplicaciones (10ma ed.). México: Pearson Educación.

Nicolás, F. L. (1663). Arte y Vso de Architectvura. Segunda parte. Madid: Petrus a Villafranca.

Ovando Grajales, F. (2013). Los [posibles] tratados de arquitectura en el equipaje de los dominicos con destino a Chiapas en el siglo XVI. Boletín de monumentos históricos(27), 151-162.

Palladio, A. (1570/2008). Los cuatro libros de arquitectura. Madrid: Akal.

Paradis, E. (2012). Anlysis of Phylogenetics and Evolutions with R. Springer. doi:https://doi. org/10.1007/978-1-4614-1743-9

Rapoport, A. (1972). Vivienda y Cultura. Barcelona: Gustavo Gili.

Serlio Bolognese, S. (1547). Quinto libro d)architettura di Sebastiano Serlio Bolognese: nel quale se tratta de diuerse forme de tempii sacri secondo il costume christiano e il mundo antico. A Paris : De 1〉Imprimerie de Michel de Vascosan. Obtenido de https:/archive.org/details/ ldpd_12029519_000/page/n5/mode/2up

Triola, M. F. (2013). Estadística. México: Pearson.

Vitruvio Polión, M. (27/1741). De Architectura. Compendio de los Diez Libros de Arquitectura (1ra ed.). (D. J. Castañeda, Trad.) Madrid: Real Academia de S. Fernando. 
Wells, M. (2010). Engineers. A history of engineering and structural design. Oxfordshire: Routledge.

Wickham, H. (2016). ggplot2 Elegant Graphics for Data Anlysis. Springer. doi:https://doi. org/10.1007/978-3-319-24277-4 